Ann. Génét. Sél. anim., I978, 10 (3), 377-390.

\title{
Paramètres génétiques des races Blonde d'Aquitaine, Charolaise et Limousine utilisées en croisement pour la production de veaux de boucherie
}

\author{
J. L. FOULLEY, F. MÉNISSIER et J. GAILLARD $(*)$ \\ avec la collaboration technique de J.J. Chambeyron \\ Station de Génétique quantitative et appliquée \\ Centre national de Recherches zootechniques, I.N.R.A., \\ 78350 Jouy-en-Josas \\ (*) Section d'Améliovation génétique \\ Institut technique de l'Élevage bovin \\ I49, rue de Bercy, 75579 Paris Cedex I2
}

\section{Résumé}

Les données du contrôle de descendance des taureaux de races à viande françaises utilisés par insémination artificielle en croisement ont été exploitées pour estimer les paramètres génétiques relatifs aux principaux caractères contrôlés en ferme dans le cadre d'une production traditionnelle de veaux de boucherie. L'analyse a porté sur 4696 , I 2284 et I 6765 veaux purs et croisés issus de 94,256 et 374 taureaux de races Blonde d'Aquitaine, Charolaise et Limousine respectivement.

Les caractères étudiés concernent la note et la fréquence des difficultés de vêlage, le poids à la naissance, le gain moyen quotidien entre la naissance et 75 jours, le poids à 75 jours, le pointage en vif de la valeur bouchère et le produit de ces deux dernières variables.

Les données ont été en un premier temps corrigées pour les effets du sexe du veau, de la race et du rang de vêlage de la mère à partir d'estimées de moindres carrés. Les cœfficients d'héritabilité ainsi que les corrélations génétiques et phénotypiques ont ensuite été estimés à partir des composantes paternelle et résiduelle des variances et covariances des caractères déduites d'une analyse des données corrigées selon un modèle aléatoire à facteurs hiérarchiques : série, taureau intra-série.

Les estimées des coefficients d'héritabilité du poids à 75 jours et du gain o-75 jours sont très homogènes entre races et ont pour valeur 0,14 et 0 , Io respectivement. Le poids à la naissance, malgré l'imprécision de sa mesure, s'avère au moins aussi héritable que le poids à 75 jours; les estimées sont de o,19; 0,1 7 et o,09 en races Blonde d'Aquitaine, Charolaise et Limousine respectivement. L'héritabilité de la conformation bouchère en vif varie selon les races paternelles : $h^{2}=0,07 ; 0,13$ et 0,29 en races Blonde d'Aquitaine, Limousine et Charolaise respectivement. Quant aux difficultés de naissance, leur héritabilité s'avère très faible $\left(0,0 \mathrm{r}<h^{2}<0,06\right)$ quelle que soit la race paternelle, mais la note de difficultés est génétiquement très liée $\left(r_{\mathbf{g}} \simeq+0,9\right)$ au poids à la naissance des veaux. Ces résultats confirment l'intérêt qu'il y a de considérer le poids à la naissance des veaux dans les critères de sélection des taureaux destinés au croisement terminal. 


\section{Introduction}

L'efficacité réelle des différentes étapes (choix sur ascendance, performances individuelles et descendance croisée) du schéma de sélection des mâles de races à viande destinés au croisement terminal a été clairement mise en évidence par FREBLING et al. (1972) et, plus récemment, par GaILLARD et al. (I974). De plus, la mise en place systématique dans toutes les séries de comparaison de mâles des différentes unités de sélection concernées d'un lot national de taureaux de référence permettra de mieux apprécier, à l'avenir, cette efficacité dans le temps et dans l'espace en tenant compte plus objectivement des différences génétiques existant entre séries de comparaisons.

L'étude théorique de ces schémas sur des bases génétiques et économiques (MocQuot et FouldEY, I973) n'en demeure pas moins nécessaire, compte tenu de l'évolution des objectifs de production et de sélection (régression de la production traditionnelle de veaux de boucherie) et des modalités de contrôle (passage du contrôle de descendance en ferme sur veaux de boucherie au contrôle de la descendance en station sur veaux de boucherie ou jeunes bovins). Dans ces études interviennent des paramètres génétiques dont les estimées malheureusement trop imprécises constituent, entre autres, un facteur limitant la portée des comparaisons des différentes options concurrentes. De meilleures estimations de ces paramètres s'avèrent indispensables notamment :

- quand on désire choisir ou parfaire certains critères de sélection tels que le choix entre sélection directe et indirecte (SYRSTAD, I970) ou l'opportunité de combiner plusieurs informations (CUNNINGHAM, I969; HILL, I974);

- dans le souci de mieux prévoir l'incidence indirecte de la sélection sur certains caractères peu ou pas considérés jusqu'à ces dernières années (croissance prénatale par exemple).

A cet égard, et faisant suite à l'étude initiale de Poujardieu et Vissac (1968) en races Charolaise et Limousine et à celle d'OrTiz MARTINEZ (I973) dans cette dernière race, il nous est apparu intéressant de faire une mise au point sur les paramètres génétiques de la croissance et de la conformation des veaux de boucherie contrôlés en ferme dans le cadre des opérations de mise à l'épreuve sur descendance des taureaux de races à viande. Cette analyse porte cette fois sur les trois principales races à viande françaises (Blonde d'Aquitaine, Charolaise et Limousine) et sur des effectifs de veaux et de descendances paternelles beaucoup plus importants. Elle doit permettre notamment d'apprécier plus précisément les répercussions à attendre sur l'évolution de la croissance prénatale des veaux et corrélativement sur celle de la fréquence des vêlages difficiles d'une sélection intense sur les critères de croissance et de conformation au sevrage. Les estimations des paramètres génétiques relatifs à ces phénomènes serviront également à définir les moyens nécessaires pour concilier au mieux dans la sélection ces objectifs de croissance au demeurant antagonistes.

\section{Matériel et méthodes}

Les données analysées proviennent du fichier de contrôle de descendance en ferme de veaux de boucherie issus de jeunes taureaux Blonds d'Aquitaine, Charolais et Limousins destinés à être utilisés en insémination artificielle, essen- 
tiellement comme races paternelles de croisement terminal. Les modalités de mise en place des inséminations et de réalisation des contrôles en ferme effectués lors de ces opérations de mises à l'épreuve sur descendance ont été décrites en détail par ailleurs (VISSAC, I964; POUJARDIEU et VISSAC, I968). Il est à rappeler simplement que ces comparaisons de jeunes reproducteurs sont faites périodiquement par chaque centre d'insémination et indépendamment pour chacun d'entre eux. Les inséminations sont réparties au hasard et pendant une période limitée dans les élevages de la zone que contrôle le centre, à raison de 300 par taureau en moyenne sur des cheptels de femelles de races diverses : laitières ou mixtes, rustiques et à viande. L'objectif est de produire une soixantaine de veaux mâles et femelles par taureau, avec un veau en moyenne par exploitation, conduits pour la production traditionnelle de veaux de boucherie allaités par leurs mères dans des conditions variables selon les régions. La répartition des veaux contrôlés selon les races paternelles et maternelles est donnée au tableau $\mathrm{I}$. Pour chacune des races de pères, ont été également inđiqués les nombres de séries et de taureaux analysés. Deux échantillons ont été considérés selon que l'information était en toute (b) ou partie (a) présente sur chacun des veaux. Compte tenu des critères de sélection effectivement appliqués à ce stade de sélection (VISSAc, 1964) et des renseignements fournis à la naissance du veau par l'éleveur, l'analyse a porté sur les variables suivantes :

- les poids des veaux à la naissance, à 75 jours (calculé par intrapolation entre deux pesées) et le gain moyen quotidien de la naissance à 75 jours;

- le pointage de conformation bouchère (somme des notes d'appréciation du développement musculaire du devant, du dessus et de l'arrière) et le produit de ce dernier et du poids à 75 jours comme critère synthétique estimateur de la valeur économique du veau;

- la note subjective des difficultés de naissance établie sur une gamme d'appréciation de difficultés croissantes de la mise bas ( $\mathrm{I}$ : vêlage facile sans assistance, $2:$ vêlage avec légère assistance de l'éleveur, $3:$ vêlage difficile, $4:$ vêlage dystocique nécessitant une intervention chirurgicale) ainsi que les fréquences des naissances difficiles (note supérieure ou égale à 3) et très difficiles (note égale à 4) des veaux.

La prise en compte de ce schéma expérimental nous a conduit à effectuer, indépendamment pour chaque race paternelle, une première correction des données pour certains facteurs de variation systématiques de milieu comme la race des mères, le numéro de vêlage et le sexe des veaux. Les résultats de l'analyse de variance par moindres carrés de ces mêmes variables (moyennes, écart-types, test de signification des effets des facteurs, estimées des effets des races de mères) ont été présentés et discutés dans une note précédente par FOULLLEY et al. (I975).

Dans cette étude, les paramètres génétiques et phénotypiques (coefficients d'héritabilité, de corrélation génétique et phénotypique) ont été calculés classiquement à partir des composantes paternelle et résiduelle des variances et covariances. Ces dernières ont été déduites des résultats d'une analyse de variance et covariance des données corrigées, suivant un modèle aléatoire hiérarchique, correspondant à la classification " série ", " taureau intra série " compte tenu du groupement des taureaux en séries contemporaines. Un intervalle de confiance approché du coefficient d'héritabilité a été calculé, comme le propose Cunningham (I969) en appliquant ici la formule exacte de GRA YBILL de l'intervalle de confiance du coefficient de corrélation intraclasse relatif à un modèle équilibré à une voie. L'écart-type 
du coefficient de corrélation génétique a été calculé par la formule de RoBERTSON (1959). L'application de cette formule relativement simple conduit à des valeurs approchées donnant un ordre de grandeur de la variabilité due à l'échantillonnage. Les estimées obtenues sont vraisemblablement sous-estimées, du moins par rapport aux valeurs fournies par la formule d'Hammond et Nicholas (r972) dont, toutefois, l'application est beaucoup moins immédiate.

\section{TABLEAU I}

Répartition des veaux selon les races parentales, le sexe, et le rang de vêlage des mères

Description of calves according to parental breeds, sex and calving parity of dams

\begin{tabular}{|c|c|c|c|c|c|c|c|c|}
\hline \multirow{2}{*}{\multicolumn{3}{|c|}{ 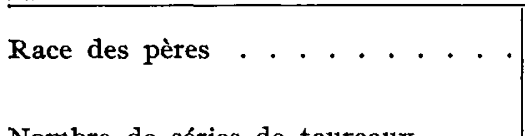 }} & \multicolumn{2}{|c|}{$\begin{array}{l}\text { Blonde } \\
\text { d'Aquitaine }\end{array}$} & \multicolumn{2}{|c|}{ Charolaise } & \multicolumn{2}{|c|}{ Limousine } \\
\hline & & & (a) & (b) & (a) & (b) & (a) & (b) \\
\hline $\begin{array}{l}\text { Nombr } \\
\text { Nombr }\end{array}$ & $\begin{array}{l}\text { e de séries } \\
\text { e total de }\end{array}$ & 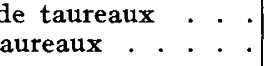 & $\begin{array}{r}7 \\
94\end{array}$ & $\begin{array}{r}5 \\
72\end{array}$ & $\begin{array}{r}15 \\
256\end{array}$ & $\begin{array}{r}13 \\
229\end{array}$ & $\begin{array}{r}\text { I3 } \\
374\end{array}$ & $\begin{array}{r}10 \\
306\end{array}$ \\
\hline \multirow{3}{*}{$\begin{array}{c}\text { Races } \\
\text { de } \\
\text { mères }\end{array}$} & $\begin{array}{l}\text { Laitières } \\
\text { et } \\
\text { mixtes }\end{array}$ & $\begin{array}{l}\text { Frisonne Française } \\
\text { Montbéliarde. } \\
\text { Pie Rouge de l'Est } \\
\text { Normande } \\
\text { Brune des Alpes }\end{array}$ & $\begin{array}{r}399 \\
27 \\
-- \\
688 \\
280\end{array}$ & $\begin{array}{r}\text { I } 084 \\
\text { I } 2 \\
\\
492 \\
\text { I } 31\end{array}$ & $\begin{array}{r}3627 \\
2060 \\
1934 \\
376 \\
33^{8}\end{array}$ & $\begin{array}{r}3128 \\
\text { I } 909 \\
\text { I } 814 \\
255 \\
237\end{array}$ & $\begin{array}{r}268 \\
266 \\
- \\
\text { I } 644 \\
677\end{array}$ & $\begin{array}{l}\text { I } 743 \\
\text { I98 } \\
- \\
\text { I } 44 \text { I } \\
455\end{array}$ \\
\hline & Rustiques & 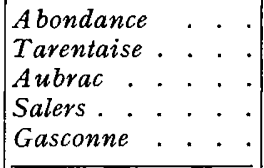 & $\begin{array}{l}- \\
\text { I6 } \\
70 \\
55\end{array}$ & $\begin{array}{r}- \\
3 \\
20 \\
22\end{array}$ & $\begin{array}{r}170 \\
53 \\
322 \\
1260 \\
212\end{array}$ & $\begin{array}{r}\text { I } 56 \\
48 \\
2 \text { I } 5 \\
833 \\
2 \text { I } 2\end{array}$ & $\begin{array}{r}60 \\
61 \\
328 \\
\text { I } 152 \\
\mathrm{I} 86\end{array}$ & $\begin{array}{r}53 \\
47 \\
224 \\
831 \\
74\end{array}$ \\
\hline & A viande & 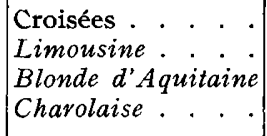 & $\begin{array}{r}323 \\
47 \\
772 \\
19\end{array}$ & $\begin{array}{r}84 \\
28 \\
\text { I I } 21 \\
\text { I } 5\end{array}$ & $\begin{array}{r}74 \mathrm{I} \\
\quad 78 \\
\overline{6}_{53}\end{array}$ & $\begin{array}{r}\text { I } 4 \text { I } \\
59 \\
- \\
595\end{array}$ & $\begin{array}{r}\text { I } 930 \\
6999 \\
599 \\
595\end{array}$ & $\begin{array}{r}\text { I } 263 \\
5628 \\
520 \\
449\end{array}$ \\
\hline Rang & de vêlage & $\begin{array}{l}\text { I }^{\mathrm{e}} \text { vêlage } \\
2^{\mathrm{e}} \text { vêlage } \\
3^{\mathrm{e}} \text { au } 6^{\mathrm{e}} \text { vêlage. } \\
7^{\mathrm{e}} \text { vêlage et plus, } \\
\text { ou inconnu }\end{array}$ & $\begin{array}{r}319 \\
278 \\
2309 \\
\quad 790\end{array}$ & $\begin{array}{r}295 \\
244 \\
825 \\
\times \quad 721\end{array}$ & $\begin{array}{r}244 \\
\text { I } 670 \\
834^{\circ} \\
2562\end{array}$ & $\begin{array}{r}212 \\
\text { I } 546 \\
6444 \\
2 \quad 286\end{array}$ & $\begin{array}{ll}2 & 032 \\
2 & 117 \\
7 & 823 \\
4 & 793\end{array}$ & $\begin{array}{l}\text { I } 602 \\
\text { I } 666 \\
5457 \\
4201\end{array}$ \\
\hline \multicolumn{2}{|c|}{ Sexe des veaux } & 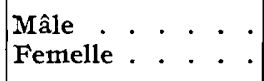 & $\begin{array}{l}243^{8} \\
225^{8}\end{array}$ & $\begin{array}{l}\text { I } 612 \\
\text { I } 500\end{array}$ & $\begin{array}{l}6676 \\
6 \times 48\end{array}$ & $\begin{array}{l}5488 \\
5000\end{array}$ & $\begin{array}{ll}9 & \text { II } 2 \\
7 & 653\end{array}$ & $\begin{array}{l}7040 \\
5886\end{array}$ \\
\hline To & tal. . & $\cdot \cdot \cdot \cdot \cdot \cdot \cdot \cdot \cdot \cdot \cdot$ & 4696 & 3 II 2 & I 2824 & Iо 488 & I6 765 & 12926 \\
\hline
\end{tabular}

(a) Ensemble des veaux contrôlés ayant servi à l'analyse.

(b) Echantillon des veaux contrôlés pour lequel toutes les variables sont présentes pour chaque veau. 


\section{Résultats et discussion}

\section{I. - Coefficients d'héritabilité}

Les estimées des coefficients d'héritabilité des différentes variables analysées sont rassemblées au tableau 2 ainsi que leur intervalle de con fiance, les composantes de la variance entre pères et résiduelle et le test $\mathrm{F}$ approché de signification des effets du père des veaux.

TABLEAU 2

Analyse de variance et estimation des coefficients d'héritabilité Analysis of variance and estimation of heritability coefficients

\begin{tabular}{|c|c|c|c|c|c|c|c|}
\hline \multirow{2}{*}{ Variable } & \multirow{2}{*}{$\begin{array}{c}\text { Race } \\
\text { de } \\
\text { père }\end{array}$} & \multirow{2}{*}{$\begin{array}{l}\text { Test } \\
\text { "F" } \\
\text { de 1'effet } \\
\text { père }\end{array}$} & \multicolumn{2}{|c|}{$\begin{array}{l}\text { Composante } \\
\text { de la variance }\end{array}$} & \multicolumn{3}{|c|}{ Coefficient d'héritabilité } \\
\hline & & & Père & $\begin{array}{l}\text { Rési- } \\
\text { duelle }\end{array}$ & Estimée & $\begin{array}{l}\text { Interva } \\
\text { confia } \\
95 \mathrm{p} .\end{array}$ & $\begin{array}{l}\text { le de } \\
\text { ce à } \\
\text { roo }\end{array}$ \\
\hline Poids à $\underset{(\mathrm{kg})}{\mathrm{la} \text { naissance }}$ & $\begin{array}{l}\mathrm{BA} \\
\mathrm{CH} \\
\mathrm{LI}\end{array}$ & $\begin{array}{l}3,33^{* *} \\
3,02^{* *} \\
1,94^{* *}\end{array}$ & $\begin{array}{l}\mathrm{I}, 72 \\
\mathrm{I}, 6 \mathrm{I} \\
0,63\end{array}$ & $\begin{array}{l}34,37 \\
37,40 \\
28,55\end{array}$ & $\begin{array}{l}0,191 \\
0,165 \\
0,086\end{array}$ & $\begin{array}{l}0,125 \\
0,127 \\
0,062\end{array}$ & $\begin{array}{l}0,287 \\
0,217 \\
0,117\end{array}$ \\
\hline $\begin{array}{llll}\text { Poids } & \text { à } & & \\
(\mathrm{kg}) & (\mathrm{a}) & \text { jours } & \end{array}$ & $\begin{array}{l}\mathrm{BA} \\
\mathrm{CH} \\
\mathrm{LI}\end{array}$ & $\begin{array}{l}2,68 * * \\
2,68 * * \\
2,58 * *\end{array}$ & $\begin{array}{r}\text { I0,26 } \\
\text { 10,88 } \\
9,29\end{array}$ & $\begin{array}{l}283,38 \\
303,93 \\
249,72\end{array}$ & $\begin{array}{l}0,140 \\
0,138 \\
0,143\end{array}$ & $\begin{array}{l}0,086 \\
0, \text { I } 04 \\
0, \text { I } 12\end{array}$ & $\begin{array}{l}0,220 \\
0,185 \\
0,184\end{array}$ \\
\hline Gain moyen quotidien ${ }_{(\mathrm{g} / \mathrm{j})}^{(\mathrm{a})}$ & $\begin{array}{l}\mathrm{BA} \\
\mathrm{CH} \\
\mathrm{LI}\end{array}$ & $\begin{array}{l}2,16 * * \\
2,22 * * \\
2,23^{* *}\end{array}$ & $\begin{array}{lll}\text { I } & 042,2 \text { I } \\
\text { I } & \text { I } 44,27 \\
\text { I } & \text { I } 4 \text { I }, 79\end{array}$ & $\left|\begin{array}{ll}41 & 848,77 \\
43 & 901,26 \\
39 & 683,75\end{array}\right|$ & $\begin{array}{l}0,097 \\
0,102 \\
0,1 \text { I } 2\end{array}$ & $\begin{array}{l}0,053 \\
0,073 \\
0,084\end{array}$ & $\begin{array}{l}0, \mathrm{I} 63 \\
0, \mathrm{I} 4 \mathrm{I} \\
0, \mathrm{I} 47\end{array}$ \\
\hline $\begin{array}{l}\text { Pointage de valeur bouchère } \\
\text { (point) }\end{array}$ & $\begin{array}{l}\mathrm{BA} \\
\mathrm{CH} \\
\mathrm{LI}\end{array}$ & $\begin{array}{l}I, 74 * * \\
4,63^{* *} \\
2,36 * *\end{array}$ & $\begin{array}{l}0,34 \\
1,54 \\
0,65\end{array}$ & $\begin{array}{l}19,96 \\
19,38 \\
20,03\end{array}$ & $\begin{array}{l}0,067 \\
0,294 \\
0,125\end{array}$ & $\begin{array}{l}0,023 \\
0,233 \\
0,093\end{array}$ & $\begin{array}{l}0,136 \\
0,372 \\
0,167\end{array}$ \\
\hline $\begin{array}{l}\text { (b) } \\
\text { Produit du poids à } 75 \text { jours } \\
\text { par le pointage (p. Ioo) }\end{array}$ & $\begin{array}{l}\mathrm{BA} \\
\mathrm{CH} \\
\mathrm{LI}\end{array}$ & $\begin{array}{l}I, 72 * * \\
3,75 * * \\
2,38 * *\end{array}$ & $\begin{array}{l}0,92 \\
3,5^{\circ} \\
1,61\end{array}$ & $\begin{array}{l}54,75 \\
58, \text { OI } \\
49,33\end{array}$ & $\begin{array}{l}0,066 \\
0,227 \\
0,127\end{array}$ & $\begin{array}{l}0,022 \\
0,176 \\
0,094\end{array}$ & $\begin{array}{l}0,134 \\
0,293 \\
0,169\end{array}$ \\
\hline $\begin{array}{l}\text { Note des conditions } \\
\text { de naissance (p. Ioo) }\end{array}$ & $\begin{array}{l}\text { BA } \\
\text { CH } \\
\text { LI }\end{array}$ & $\begin{array}{l}\mathrm{I}, 50^{* *} \\
\mathrm{I}, 53^{* *} \\
\mathrm{I}, 23^{* *}\end{array}$ & $\begin{array}{l}43,32 \\
34,14 \\
16,33\end{array}$ & $\begin{array}{ll}3 & 995,22 \\
3 & 047,48 \\
3 & 038,71\end{array}$ & $\begin{array}{l}0,043 \\
0,044 \\
0,022\end{array}$ & $\begin{array}{l}0,011 \\
0,024 \\
0,004\end{array}$ & $\begin{array}{l}0,090 \\
0,072 \\
0,040\end{array}$ \\
\hline $\begin{array}{l}\text { Fréquence des naissances } \\
\text { difficiles (p. I0o) }\end{array}$ & $\begin{array}{l}\mathrm{BA} \\
\mathrm{CH} \\
\mathrm{LI}\end{array}$ & $\begin{array}{l}\mathrm{I}, 25^{* *} \\
\mathrm{I}, 72^{* *} \\
\mathrm{I}, 2 \mathrm{I} *\end{array}$ & $\begin{array}{r}12,30 \\
25,83 \\
8,35\end{array}$ & $\begin{array}{l}2304,83 \\
\text { I } 696,2 \mathrm{I} \\
\text { I } 667,05\end{array}$ & $\begin{array}{l}0,02 I \\
0,060 \\
0,020\end{array}$ & $\begin{array}{r}0,001 \\
0,037 \\
0,004\end{array}$ & $\begin{array}{l}0,061 \\
0,090 \\
0,040\end{array}$ \\
\hline $\begin{array}{l}\text { (a) } \\
\text { Fréquence des naissances } \\
\text { très difficiles (p. roo) }\end{array}$ & $\begin{array}{l}\text { BA } \\
\text { CH } \\
\text { LI }\end{array}$ & $\begin{array}{l}\mathrm{I}, 3 \mathrm{O}^{* *} \\
\mathrm{I}, 25^{* *} \\
\mathrm{I}, \mathrm{I} 4^{*}\end{array}$ & $\begin{array}{l}4,30 \\
\mathrm{I}, 89 \\
\mathrm{I}, \mathrm{I} 3\end{array}$ & $\begin{array}{l}67 \mathrm{I}, 6 \mathrm{I} \\
360,67 \\
333,6 \mathrm{I}\end{array}$ & $\begin{array}{l}0,025 \\
0,021 \\
0,014\end{array}$ & $\begin{array}{r}-0,002 \\
0,004 \\
-0,002\end{array}$ & $\begin{array}{l}0,067 \\
0,044 \\
0,036\end{array}$ \\
\hline
\end{tabular}

$*: \mathbf{P}<0,05$

** : $\mathbf{P}<0$,or. bles sont présentes.

$\mathbf{B A}=$ Blonde d'Aquitaine, $\mathbf{C H}=$ Charolaise, $\mathbf{L I}=$ Limousine. (a) Analyse effectuée sur l'ensemble des veaux contrôlés.

(b) Analyse effectuée sur l'échantillon des veaux pour lesquels toutes les varia- 
D'une façon générale, les estimées obtenues sont faibles $(0,02$ à 0,29$)$ quels que soient le caractère et la race paternelle envisagés eu égard à l'hétérogénéité des conditions d'élevage en ferme et à l'imprécision de l'information recueillie. Les estimées sont beaucoup plus faibles pour les critères d'appréciation des difficultés de naissance (effet direct du père) $\left(h^{2}=0,02\right.$ à 0,06$)$ que pour les critères de croissance $\left(h^{2}=0\right.$, Io à 0,19$)$, de conformation et valeur synthétique bouchères $\left(h^{2}=0,07\right.$ à 0,29$)$.

Les coefficients d'héritabilité du poids à la naissance sont très similaires en race Blonde d'Aquitaine $\left(h^{2}=0, \mathrm{I} 9\right)$ et Charolaise $\left(h^{2}=0, \mathrm{I} 7\right)$ et supérieurs à $1 \mathrm{a}$ valeur calculée en race Limousine $\left(h^{2}=0,09\right)$. Cette différence est due essentiellement à une variabilité entre taureaux nettement moindre dans cette race comme en attestent les valeurs comparées des composantes de la variance entre pères et intrapères. PoIVEy (I973), sur un fichier plus restreint et avec une méthode de calcul de l'héritabilité moins précise, avait relevé le même phénomène avec toutefois des différences d'héritabilité plus accusées entre races Charolaise $\left(h^{2}\right.$ $=0,26)$ et Blonde d'Aquitaine $\left(h^{2}=0,27\right)$ d'une part et Limousine $\left(h^{2}=0,09\right)$ d'autre part.

A partir de ce même fichier de contrôle de descendance analysé en I 968 , PoujARDIEU et VISSAC trouvaient des valeurs de 1'héritabilité du poids à la naissance et des composantes de la variance entre pères et résiduelle respectivement de 0,$24 ; 2,62$ et 4r,54 en Charolais et de 0,$19 ; 1,42$ et 29,73 en Limousin; ces dernières valeurs sont comparables à ce qu'on observe maintenant dans les races Blonde d'Aquitaine et Charolaise. Tous ces résultats laissent entrevoir la possibilité d'une réduction de variabilité génétique du poids à la naissance principalement en race Limousine. Cette réduction pourrait être due aux diverses sélections pratiquées avant la mise en contrôle de descendance, en particulier à celles effectuées sur ascendance et à 1 'issue du contrôle individuel et dont l'efficacité a été clairement démontrée (GAILLARD et al., I974).

Les coefficients d'héritabilité du poids à 75 jours et de la croissance entre la naissance et 75 jours sont très homogènes entre les trois races paternelles : $h^{2}=0,14$ et o, Io respectivement. En race Blonde d'A quitaine, la légère réduction d'héritabilité observée entre la naissance et 75 jours $\left(h^{2}=0\right.$, I9 puis 0,14$)$, bien que vraisemblablement non significative, va dans le sens des conclusions de la plupart des auteurs qui ont étudié les variations d'héritabilité du poids au cours de la vie de l'animal. Cette diminution du coefficient d'héritabilité pendant la phase d'allaitement et de sevrage ne s'observe pas sur les croisés Charolais et Limousins contrairement à ce que trouvaient POUJARDIEU et VISSAC (I g68) dans la première race $\left(h^{2}=0,24\right.$ et 0,06 respectivement à la naissance et à 75 jours) et MoLINUEvo et VISSAC (I972) dans les élevages de sélection de ces 2 races.

Il est intéressant de comparer ces résultats par rapport à l'ensemble des estimations bibliographiques relatives à la croissance jusqu'au sevrage obtenues à l'étranger sur des populations spécialisées pour la production de viande, de races anglo-saxonnes essentiellement, et conduites dans des conditions plus extensives. Les principales synthèses bibliographiques effectuées à ce sujet, dont celles de PetTty et Cartwright (I966), Preston et Wilitis (I970) et Ortiz Martinez (I973) indiquent des estimées moyennes des coefficients d'héritabilité du poids à la naissance respectivement pour chacun des auteurs de 0,$44 ; 0,38$ et 0,30 , du poids au sevrage de 0,$32 ; 0,30$ et 0,32 et du gain de poids de la naissance au sevrage (6 à 8 mois) de 0,$34 ; 0,27$ et 0,28 , s'avérant nettement supérieures aux nôtres. Une telle discordance peut s'expliquer en tenant compte : 
- de ce que les estimées obtenues à l'étranger sont calculées généralement sur des données plus finement corrigées, en particulier souvent pour l'effet du troupeau;

- qu'il s'agit de moyennes de valeurs s'étalant sur une grande gamme de distribution (ORTIZ MARTINEZ, I973);

- que ces études portent sur un échantillon de taureaux beaucoup moins sélectionné que le nôtre;

- qu'enfin, on observe généralement un accroissement de l'héritabilité des poids de 3-4 mois à 6-8 mois, âge où du fait de la complémentation, l'influence maternelle est moins déterminante dans l'expression du potentiel de croissance.

Le coefficient d'héritabilité du pointage de conformation bouchère diffère sensiblement d'un type de croisement à l'autre et se trouve supérieur chez les croisés Charolais $\left(h^{2}=0,29\right)$ aux estimées des croisés Limousins $\left(h^{2}=0,12\right)$ et Blonds d'Aquitaine $\left(h^{2}=0,07\right)$. On retrouve ainsi la différence entre croisés Charolais et Limousins mise en évidence précédemment par PouJARDIEU et VISSAC (I968), ces auteurs indiquant des estimations de 0,32 et 0,16 respectivement en Charolais et Limousin. Cette hétérogénéité traduit vraisemblablement l'incidence du mode de choix et de l'intensité de celui effectué sur la conformation avant la mise à l'épreuve sur descendance dans chacune des races. Les choix sur descendance et à l'issue du contrôle individuel ont, en effet, été pratiqués plus tôt et plus systématiquement en race Blonde d'Aquitaine et à un moindre degré en race Limousine qu'en race Charolaise. L'héritabilité du produit du poids à 75 jours par le pointage, proposé par VISSAC (1964) comme critère d'indice synthétique de valeur bouchère, montre la même hétérogénéité que celle relevée pour le pointage, la variabilité génétique du poids à 75 jours ne différant pas d'une race à l'autre.

Par rapport aux valeurs de l'héritabilité de la conformation bouchère calculées ici, les estimées bibliographiques moyennes relatives au pointage au sevrage de races anglo-saxonnes sont légèrement supérieures à celle trouvée ici en Charolais; ces valeurs sont de 0,$36 ; 0,32$ et 0,35 respectivement selon PETTY et CARTWRIGHT (I966), Preston et Wiliris (I970) et Ortiz Martinez (I973).

En ce qui concerne les difficultés de naissance, l'étude a trait uniquement à l'effet direct du père sur ce caractère qui est le reflet de l'influence des gènes transmis par le père sur la taille et la morphologie du veau qu'il engendre, conformément à la décomposition de MENISSIER (I974).

Les estimations obtenues, qu'elles aient trait à la note de difficultés de naissance, à la fréquence des naissances difficiles ou à celle des naissances très difficiles sont très faibles (de I à $6 \mathrm{p}$. Ioo). Ces valeurs ne permettent pas de déceler des différences statistiquement significatives de variabilité génétique entre races malgré une certaine tendance logique à une variabilité plus marquée en Charolais qu'en Limousin. Ces résultats sont tout à fait comparables à ceux trouvés par BELIC et MENISSIER (I968) en croisement pour la race Charolaise et par BRINKs et al. (r973) en Hereford dans le cas, il est vrai plus complexe, des performances en race pure. Les premiers en utilisant la transformation arcsin $\sqrt{ }$ sur la fréquence des naissances assistées de 73 descendances paternelles et le second, la méthode conventionnelle de LuSH et al. (I948), sur 2733 veaux Hereford issus de 223 pères, trouvent des valeurs du coefficient d'héritabilité respectivement de 0,048 d'une part, $0,069 \pm 0,022$ et $0, \mathrm{I} 26 \pm 0$, Iog d'autre part selon que tous les veaux sont considérés ou seulement ceux de vêlages à 2 ans. 
Sans entrer plus avant dans la discussion de ces paramètres génétiques et de leur utilisation à des fins de sélection, sujet qui a été très bien synthétisé dans un récent rapport C.E.E. (PHILIPSson, I978), ces résultats laissent à penser qu'une sélection des mâles sur les seules notes de difficultés de naissance de leurs veaux (issus de mères de tous âges) sera relativement peu efficace. Sinon il faut alors contrôler un grand nombre de descendants par taureau (LINDHE, I974). On peut, en effet, calculer qu'il faut I85 descendants contrôlés par taureau pour obtenir une précision $\left(R^{2}\right)$ de la valeur génétique de 0,7 en supposant un coefficient d'héritabilité de 5 p. Ioo.

\section{2. - Corrélations génétiques et phénotypiques (tabl. 3)}

Les coefficients de corrélation génétique sont en général supérieurs aux coefficients de corrélation phénotypique. Comme l'indique FALCONER (I960), les caractères en jeu ayant une faible héritabilité, la corrélation phénotypique $\left(r_{p}\right)$ est

TABLEAU 3

Estimées des coefficients de corrélation génétique et phénotypique Estimations of genetic and phenotypic correlation coefficients

\begin{tabular}{|c|c|c|c|c|c|c|}
\hline \multirow[b]{2}{*}{ Caractères } & \multirow{2}{*}{$\begin{array}{c}\text { Races } \\
\text { pater- } \\
\text { nelles } \\
\left({ }^{8}\right)\end{array}$} & \multicolumn{5}{|c|}{ Corrélations avec les caractères de $(b)$} \\
\hline & & $\begin{array}{c}\text { Poids } \\
\text { à la } \\
\text { naissance }\end{array}$ & $\begin{array}{ll} & \text { Poids } \\
\text { à } & 75 \text { jours }\end{array}$ & $\begin{array}{c}\text { Gain } \\
\text { moyen } \\
\text { quotidien } \\
\left(\begin{array}{llll}0 & \text { à } & 75 & \mathrm{j}\end{array}\right)\end{array}$ & $\begin{array}{l}\text { Pointage } \\
\text { de valeur } \\
\text { bouchère }\end{array}$ & $\begin{array}{c}\text { Note de } \\
\text { difficultés } \\
\text { de naissance }\end{array}$ \\
\hline $\begin{array}{l}\text { Poids } \\
\text { à la } \\
\text { naissance }\end{array}$ & $\begin{array}{l}\text { BA } \\
\text { CH } \\
\text { LI }\end{array}$ & & $\begin{array}{ll}0,666 & (0,107) \\
0,689 & (0,056) \\
0,726 & (0,053)\end{array}$ & $\left|\begin{array}{ll}0,335 & (0,183) \\
0,373 & (0,098) \\
0,568 & (0,081)\end{array}\right|$ & $\begin{array}{ll}0,138 & (0,222) \\
0,202 & (0,086) \\
0,286 & (0,104)\end{array}$ & $\begin{array}{ll}0,885 & (0,055) \\
0,91 \text { I } & (0,024) \\
0,91 \text { I } & (0,034)\end{array}$ \\
\hline $\begin{array}{c}\text { Poids } \\
\text { à } \\
75 \text { jours }\end{array}$ & $\begin{array}{l}\mathrm{BA} \\
\mathrm{CH} \\
\mathrm{LI}\end{array}$ & $\begin{array}{l}0,408 \\
0,444 \\
0,319\end{array}$ & & $\begin{array}{ll}0,926 & (0,03 I) \\
0,930 & (0,016) \\
0,978 & (0,004)\end{array}$ & $\mid \begin{array}{ll}0,254 & (0,223) \\
0,391 & (0,081) \\
0,454 & (0,077)\end{array}$ & $\begin{array}{ll}0,319 & (0,240) \\
0,634 & (0,0877) \\
0,680 & (0,093) \\
\end{array}$ \\
\hline 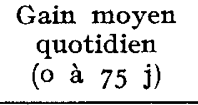 & $\begin{array}{l}\text { BA } \\
\text { CH } \\
\text { LI }\end{array}$ & $\begin{array}{r}0,057 \\
0,103 \\
-0,018 \\
\end{array}$ & $\begin{array}{l}0,936 \\
0,937 \\
0,942\end{array}$ & & $\begin{array}{ll}0,251 & (0,240) \\
0,398 & (0,086) \\
0,458 & (0,08 \mathrm{I})\end{array}$ & $\begin{array}{ll}0,046 & (0,287) \\
0,349 & (0,070) \\
0,540 & (0,130)\end{array}$ \\
\hline $\begin{array}{l}\text { Pointage } \\
\text { de valeur } \\
\text { bouchère }\end{array}$ & $\begin{array}{l}\text { BA } \\
\text { CH } \\
\text { LI }\end{array}$ & $\begin{array}{l}0,196 \\
0,246 \\
0,137\end{array}$ & $\begin{array}{l}0,514 \\
0,526 \\
0,498\end{array}$ & $\begin{array}{l}0,487 \\
0,488 \\
0,478\end{array}$ & & $\begin{array}{ll}0,572 & (0,173) \\
0,155 & (0,120) \\
0,304 & (0,159) \\
\end{array}$ \\
\hline $\begin{array}{l}\text { Note } \\
\text { de difficultés } \\
\text { de naissance }\end{array}$ & $\begin{array}{l}\text { BA } \\
\text { CH } \\
\text { LI }\end{array}$ & $\begin{array}{l}0,170 \\
0,316 \\
0,109\end{array}$ & $\begin{array}{l}0,059 \\
0,144 \\
0,067\end{array}$ & $\begin{array}{r}-0,001 \\
0,036 \\
0,032\end{array}$ & $\begin{array}{l}0,074 \\
0,134 \\
0,067\end{array}$ & \\
\hline
\end{tabular}

(a) $\mathbf{B A}=$ Blonde d'Aquitaine, $\mathbf{C H}=$ Charolaise, $\mathrm{I}_{\mathbf{I}} \mathrm{I}=$ Limousine.

(b) Au-dessus de la diagonale = corrélation génétique et écart-type; au-dessous de la diagonale = corrélation phénotypique. 
essentiellement conditionnée par la corrélation entre effets de milieu agissant simultanément sur chacun des caractères $\left(r_{\mathrm{E}}\right)$. On conçoit ainsi, à travers la diversité des conditions d'élevage des mères, de la conduite des veaux (âge et poids de vente, importance de l'alimentation non lactée complémentaire), des erreurs d'estimation du poids à la naissance donnée par l'éleveur ainsi qu'à travers la diversité de ses réactions vis-à-vis des mises bas difficiles, qu'il ne se dégage pas de liaisons individuelles très marquées entre le poids à la naissance d'une part, le poids à 75 jours et le gain de poids d'autre part, ainsi qu'entre les conditions de naissance et l'ensemble des critères. Pour ce qui est du pointage, au contraire, l'appréciation subjective faite par les experts sur un veau donné est fortement in fluencée par le poids de l'animal au moment du jugement d'où des liaisons de milieu et phénotypiques appréciables entre ces deux variables ainsi qu'indirectement entre le pointage et les poids à 75 jours ou le gain de poids.

Cette observation générale étant faite, nous analyserons successivement les liaisons entre critères de croissance, entre le pointage et les critères de croissance, entre la note de difficultés de naissance d'une part et les critères de croissance et de conformation d'autre part. Par raison de clarté, nous avons écarté de cette analyse certaines variables redondantes (fréquences de naissances difficiles et très difficiles par rapport à la note) ou induisant des corrélations automatiques (produit du poids à 75 jours et du pointage).

\section{a. Corrélations entre critères de croissance}

Les coefficients de corrélation génétique entre le poids à la naissance et le poids à 75 jours sont élevés et homogènes entre races : $r_{g}=0,67 ; 0,69$ et 0,73 respectivement chez les veaux croisés Blonds d'Aquitaine, Charolais et Limousins.

Ces valeurs sont comparables à celles obtenues par PoIvey (I973) $\left(r_{g}=0,63\right.$; o,64 et 0,5I respectivement chez les croisés Blonds d'Aquitaine, Charolais et Limousins), par POUJARDIEU et VISSAC (I968) $\left(r_{g}=0,79\right.$ et 0,68 dans les deux derniers types de croisements) et par Molinuevo et Vissac (I972) dans les élevages de sélection Charolais $\left(r_{g}=0,6 \mathrm{I}\right.$ et $0,8 \mathrm{I}$ chez les veaux mâles et femelles) et Limousins $\left(r_{g}=0,77\right.$ chez les veaux mâles). ORTIZ MARTINEz (I973) obtenait au contraire sur des veaux de boucherie Limousins purs et croisés des valeurs plus faibles comprises entre 0,30 et 0,50 . Les coefficients de corrélation génétique et phénotypique entre le poids à la naissance et la croissance de la naissance au sevrage sont logiquement plus faibles que ceux entre les poids correspondants du fait de la liaison algébrique existant entre les deux variables. La liaison génétique demeure toutefois nettement positive. Cette liaison génétique entre croissance pré et postnatale tient selon TAYLOR (1968) à l'intervention dans le processus de croissance d'un grand nombre de gènes identiques tout au long de la vie de l'animal. Elle implique l'existence d'une réponse corrélative sur le poids à la naissance même par sélection sur la croissance postnatale.

Les résultats de PetTry et CARTwRIght (I966) indiquent globalement les mêmes tendances que celles relevées ici. Ces auteurs donnent des valeurs pondérées des coefficients de corrélations génétique et phénotypique de $r_{g}=0,5^{8}$ et $r_{p}=0,39$ et de $r_{g}=0,38$ et $r_{p}=0,23$ entre d'une part le poids à la naissance, d'autre part le poids au sevrage, et la croissance de la naissance au sevrage; ces valeurs sont très comparables aux nôtres compte tenu des différences d'âge pour le poids postnatal. 


\section{b. Liaison entre critères de croissance et de conformation}

Les coefficients de corrélation génétique et phénotypique entre le poids à la naissance et le pointage de conformation bouchère sont faibles $\left(r_{g}=0,138 ; 0,202\right.$ et 0,286 respectivement en race Blonde d'Aquitaine, Charolaise et Limousine); la liaison s'avère, toutefois, plus marquée chez les veaux croisés Limousins à la différence de ce que trouvaient POUJARDIEU et VISSAC (I968) $\left(r_{g}=0,06\right)$; ORTJZ MARTINEZ (I973) a fourni des valeurs intermédiaires $\left(r_{g}=0, \mathrm{I} 7\right.$ chez les veaux mâles et 0,23 chez les veaux femelles).

Le pointage de conformation bouchère effectué vers 2 mois et demi, est très dépendant génétiquement du poids à 75 jours, surtout chez les veaux croisés Charolais et Limousins $\left(r_{g}=0,39\right.$ et 0,45 respectivement), conformément aux valeurs estimées par PoujARdieu et Vissac (I968) et OrTiz MARTINEZ (1973) : $r_{g}=0,46$ et 0,34 en Charolais et Limousin pour les premiers auteurs, $r_{g}=0,37$ et 0,43 sur des veaux croisés Limousins mâles et femelles pour le dernier auteur. La valeur plus faible trouvée en race Blonde d'Aquitaine pour la corrélation génétique $\left(r_{g}=0,254\right)$ ne peut être attribuée à un mode d'appréciation différent et plus indépendant du poids en ce qui concerne la conformation bouchère dans cette race, mais pourrait peut-être résulter de ce que la variance entre taureaux pour le pointage étant faible, la covariance entre taureaux avec le poids se trouve estimée avec beaucoup plus d'imprécision que dans les autres races.

En races anglo-saxonnes, PETTY et CARTWRIGHT (1966) font état de coefficients moyens de corrélation génétique et phénotypique entre le pointage au sevrage d'une part, le poids à la naissance et le poids au sevrage d'autre part, respectivement de $r_{g}=0,36 ; r_{p}=0$, I5 et $r_{g}=0,39 ; r_{p}=0,39$. Les estimations de $\mathrm{KoCH}$ et CLARK (I955), BRINKS et al. (I962, r964) sont plus proches des nôtres que ne le sont les précédentes $r_{g}=0, \mathrm{I} 9 ; 0,13$ et $0, I_{3}$ respectivement). Quant aux résultats concernant le poids au sevrage, la moyenne donnée par PETTY et CARTWRIGHT masque en fait une très grande variabilité dans les estimations; PRESTON et WILLIS (I970) en présentent une gamme allant de $-0,52$ à $+0,72$. Cette disparité est à relier à la diversité de la notion de pointage selon les régions, les races et les types de productions, et aux parts respectives avec lesquelles interviennent dans le jugement, des aptitudes aussi différentes que " le développement " et "l'état ".

\section{c. Liaison entre les difficultés de naissance et les critères decroissance ouconformation:}

Phénotypiquement, la note d'appréciation des difficultés de naissance (effet direct) dépend très faiblement du poids à la naissance $\left(r_{p}=0, \mathrm{I} 7 ; 0,32\right.$ et $0, \mathrm{II}$ respectivement chez les veaux croisés Blonds, Charolais et Limousins), en raison de l'importance dans cette étude des facteurs de milieu non contrôlés.

Dans des conditions plus standardisées, le poids à la naissance s'avère le facteur causal le plus important du moins chez les primipares (MENISSIER, I974). Ainsi, sur deux échantillons de femelles contrôlées dans des conditions relativement homogènes (station de contrôle des descendances femelles de la race Charolaise, 8 élevages de sélection), COUTEAUDIER et al. (I97I) trouvent des corrélations phénotypiques entre la note de difficultés de vêlage et le poids à la naissance de 0,75 ; 0,64 et 0,24 sur des femelles respectivement primipares de 2 ans, 3 ans et sur des vaches de 4 ans et plus. Dans une expérience de croisement entre taureaux $C$ harolais et Blonds sur 47 génisses Aubrac, croisées Charolaises et Blondes d'une part et 83 génisses Gasconnes, croisées Charolaises et Blondes, Charolaises et Blondes pures, ABDAL, AH (I97I) indique des corrélations phénotypiques de 0,65 et 0,43 respec- 
tivement sur chacun des échantillons. Enfin, SEITZ (I972), sur II5 génisses vêlant à 2 ans des races Maine-Anjou, Charolaise et Limousine accouplées aux trois mêmes races de pères selon un schéma factoriel, obtient un coefficient de corrélation de 0,79 entre le poids à la naissance des veaux et la note de difficulté de vêlage de leurs mères.

Génétiquement, par contre, les liaisons entre les conditions de naissance et les critères de croissance ou de conformation sont beaucoup plus élevées qu'elles ne l'étaient phénotypiquement, d'où leur intérêt en sélection. Ainsi, le coefficient de corrélation génétique entre le poids à la naissance et la note de leurs conditions de naissance est très élevé et homogène $\left(r_{g}=0,9\right)$ entre les trois races paternelles. Ces résultats confirment parfaitement les estimations obtenues par BELIC et MENISSIER (I968) sur un échantillon plus restreint de 4507 veaux croisés issus de 73 taureaux Charolais. Dans cette étude, ces auteurs estimaient à $+0,64$ le coefficient de corrélation entre taureaux reliant le taux de naissances assistées au poids moyen des veaux à la naissance, et en déduisaient un coefficient de corrélation génétique supérieur à $\mathrm{I}$. Le pointage de conformation bouchère et le poids à 75 jours présentent eux aussi une corrélation génétique non négligeable avec la note des conditions de naissance, ce qui laisse prévoir que la sélection des taureaux sur descendance classiquement pratiquée sur ces deux critères aura une incidence indirecte défavorable sur les conditions de vêlage. Celle-ci pourrait être atténuée en partie par la substitution du gain postnatal au poids à 75 jours comme critère de sélection avec, au vu des coefficients, une meilleure efficacité en race Blonde $d^{\prime}$ Aquitaine que dans les races Charolaise et Limousine. Dans ces deux dernières, l'obtention d'une réponse indirecte espérée nulle sur la composante directe des conditions de vêlage exigerait de pénaliser davantage le poids à la naissance dans le critère de sélection pondérale (FOULLEY et ROUVIER, I97I; FoulíEY et MoliNUEVO, I97I; FOULLEY, I976).

\section{Conclusion}

Cette étude a donc permis de préciser les estimations des paramètres génétiques concernant les effets génétiques directs sur la croissance pondérale, la conformation musculaire et les difficultés de naissance dans les principales races à viande françaises. Cette étude constitue en fait un bilan de la variabilité génétique telle qu'elle s'est manifestée en moyenne sur une dizaine d'années dans le cadre des opérations de contrôle de descendance en ferme des taureaux d'I.A. utilisés pour une production de veaux de boucherie purs et croisés. L'avantage d'un traitement global ne doit pas toutefais masquer les limitations concomitantes; en l'occurrence, celles inhérentes au fait qu'on travaille sur des populations sélectionnées selon des modalités de choix des taureaux avant contrôle de descendance qui ont évolué dans le temps et ont pu différer selon les races.

L'analyse des résultats obtenus confirme généralement les principales tendances ressortant des études partielles antérieures, effectuées sur des fichiers analogues (Poujardieu et Vissac, I968; Beilic et Ménissier, ig68; Ortiz Martinez, I973; Polvey, I973) à savoir l'existence :

- d'un coefficient d'héritabilité du poids légèrement plus élevé à la naissance qu'à 75 jours en dépit de 1'appréciation moins précise de ce premier;

- d'un coefficient de corrélation génétique appréciable $\left(r_{g}=0,7\right)$ entre ces 
deux poids ainsi qu'entre le poids à la naissance et la composante paternelle des difficultés de vêlage $\left(r_{g}=0,9\right)$; ceci implique une réponse corrélative non négligeable sur le poids à la naissance et sur les difficultés de mise bas du fait de la sélection sur le poids à 75 jours.

Notre étude fait apparaître, toutefois, des valeurs du coefficient d'héritabilité du poids à la naissance généralement plus faibles que celles trouvées par ailleurs, notamment en race Limousine. De plus, nous n'observons plus la très nette différence de variabilité génétique mise en évidence par POUJARDIEU et VISSAC (I968) entre Charolais et Limousin pour le poids à 75 jours; les estimées de l'héritabilité s'avérant dans cette étude relativement homogènes.

En fin, la très forte liaison génétique existant entre la composante paternelle des difficultés de vêlage et le poids à la naissance des veaux jointe à un meilleur coefficient d'héritabilité de ce dernier critère, conduit à souligner l'intérêt de prendre en compte ce poids dans les critères de sélection des taureaux destinés au croisement terminal.

Rę̧u pour publication en décembre 1978 .

\section{Summary}

Genetic parameters of Blond d'Aquitaine, Charolais and Limousin breeds used as sire breeds for veal calf production

Progeny-test data from A.I. sires of French beef breeds used in crossbreeding were analysed for estimating the genetic parameters of the main field recorded traits concerning a traditional veal calf production. The analysis concerned 4696,12284 and 16765 purebred and crossbred offsprings of 94,256 and 374 sires from the breeds Blond d'Aquitaine, Charolais and Limousin, respectively.

The traits studied involved score and frequency of calving difficulties, birth weight, average daily gain between birth and 75 days, 75 -day weight, live conformation score and the product of the last two variables.

The data were primarily adjusted for calf sex as well as dam breed and calving parity effects by means of the least squares method. Heritability as well as genetic and phenotypic coefficients were estimated from paternal and residual components of variances and covariances of the traits derived from an analysis of adjusted data according to a nested classification of random factors (group; bull within group).

Heritability coefficient estimates of 75 -day weight (o.14) and 0-75 day gain (o.ro) were very homogenous between breeds. The birth weight, despite the inaccuracy of its measurement, proved to be at least as heritable as the 75-day weight; the $h^{2}$ estimates were $0.19,0.17$ and 0.09 , respectively for the breeds Blond d'Aquitaine, Charolais and Limousin. Heritability of live conformation score varied according to paternal breeds: $h^{2}=0.07 ; 0.13$ and 0.29 for the breeds Blond d'Aquitaine, Limousin and Charolais, respectively. Heritability of calving difficulties appeared to be very low (o.or $<h^{2}<0.06$ ) whatever the paternal breed, but the score was genetically highly correlated $\left(r_{\mathrm{g}} \simeq+0.9\right)$ with the birth weight of the calves. These results confirm the interest of considering the birth weight of the calves in the selection criteria of the sires intended for terminal crossing.

\section{Références bibliographiques}

ABDallah O. Y., I97I. Variations génétiques de l'aptitude au vêlage et de ses composantes. Thèse doct. Ing., Fac. Sci. Paris, 300 p.

BELIC M., MENissier F., I968. Étude de quelques facteurs influençant les difficultés de vêlage en croisement industriel. Ann. Zootech., 17, ro7-142. 
Brinks J. S., Olson J. E., Carol, E. J., 1973. Calving difficulty and its association with subsequent productivity in Hevefords. J. Anim. Sci., 36, I I-I 7.

Couteaudier J. F., Regis R., Ménissrer F., r97I. Possibilités de sélection de l'aptitude au vêlage en race Charolaise. $\mathrm{X}^{\mathrm{e}}$ Congr. Int. Zootech., Féd. Europ. Zootech., Paris-Versailles, I 7-I9 juillet I97 I, Io $\mathrm{p}$.

Cunningham E. P., r969. Animal Breeding Theory. Landbruksbokhandeen/Universistet sforlaget, Vollebekk/Oslo.

FALCONER D. S., I96o. Introduction to Quantitative Genetics. Oliver et Boyd, London, p. 365.

FOULLEY J. L., 1976. Some considerations on selection criteria and optimization for terminal sire breeds. Ann. Génét. Sél. anim., 8, 89-ror.

Foul. IEy J. L., Rouvier R., I97I. Méthode d'établissement des index de sélection des taureaux de races à viande sur la valeur bouchère de leurs descendants. Ann. Génét. Sél. anim., 3, 497-507.

Fould,ey J. L., Molrnuevo H. A., I971. Possibilité de modification par sélection de la forme de la courbe de croissance avant sevrage des veaux de vaces à viande françaises. $\mathrm{X}$ Congr. Int. Zootech., Paris-Versailles, I 7-19 juillet, 6 p.

Foulley J. L., Ménissier F., Gaillard J., Nebreda A. M., 1975. Aptitudes maternelles des races laitières, mixtes, rustiques et à viande pour la production de veaux de boucherie. Livest. Prod. Sci., 2, 39-49.

Frebling J., Gaillard J., Vissac B., I971. Dix ans de choix et d'utilisation par insémination artificielle de taureaux de races à viande pour la production de veaux de boucherie. $\mathrm{X}$ Congr. Int. Zootech., Féd., Europ. Zootech., Paris-Versailles, I7-I9 juillet, 20 p.

Gailitard J., Foulley J. L., MÉnissier F., I974. Observations sur l'efficacité du choix sur ascendance paternelle et performances individuelles de taureaux de races à viande destinés au croisement terminal. I er Congr. Mond. Génét. appl. Elev. anim., Madrid, 7-I I octobre I974, III, $877-887$.

HAMmOND K., Nicholas F. W., I972. The sampling variance of the correlation cofficients estimated from two-fold nested and offspring-parent resgression analyses. Theoret Appl. Genet., 42, 97-100.

HILL W. G., I974. Heritabilities: estimation problems and the present state of information. $I^{\text {er }}$ Congr. Mond. Génét. Appl. Elev. anim., Madrid, 7-I I octobre I974, I, 343-35I.

Koch R. M., ClaRk R. T., I955. Genetic and environmental relationships among economic characters in beef cattle. $J$. anim. Sci., 14, 775-791; 979-996.

Lindhe B., I974. Improvement in beef-breeding by selection. I $^{\text {er }}$ Congr. Mond. Génét. Appl. Elev. anim., Madrid, 7-II octobre I974, I, 655-669.

I,USH J. L., LAMOREUX W. F., HAzEL L. N., I948. The heritability of resistance to death in the Fool. Poult. Sci., 27, 375-388.

Molinuevo H. A., Vissac B., I972. Variabilité génétique de la croissance avant sevrage dans les races Charolaise et Limousine. Ann. Génét. Sél. anim., 4, 423-444.

MénISSIER F., r974. L'aptitude au vêlage des races à viande françaises : 1'origine des difficultés de vêlage et leur amélioration génétique. XIe journées d'information du "Grenier de Theix "; "L'exploitation des troupeaux de vaches allaitantes", 25-30 novembre I973. Theix, Bull. Tech., C.R.Z.V.-I.N.R.A., oct. 74, I39-I 70.

Mocquot J. C., Foullex J. L., I973. Recherche des conditions de rentabilité d'un schéma de sélection d'une souche de bovins destinée au croisement de première génération pour la production de veaux de boucherie. Ann. Génét. Sél. anim., 5, r89-209.

Ortiz Martinez A., I973. Efficacité des méthodes de choix des taureaux d'insémination artificielle en race Limousine. Thèse doct. Ing. Sci. nat., Fac. Sci., Paris VI, I 20 p.

PETTY R. B. Jr., CARTWRight T. C., I966. A summary of genetic and environmental statistics for growth and conformation traits of young beef cattle. Depart. Tech. Rep. $\mathrm{n}^{\circ}$, Texas A et M University, College Station, Texas, $54 \mathrm{p}$.

Phil.ipsson J., Foulley J. L., Lederer J., Liboriussen T., Osinga A., 1978. Sire evaluation and breeding strategies for limiting dystocia and stillbirth. Livest. Prod. Sci., (submitted).

Porvey J. P., I973. Etude des liaisons entre les effets paternels sur la croissance prénatale et la croissance postnatale chez les bovins à viande. Dipl. Et. appr. Génét. Quant. appli., Fac. Sci., Paris VI, $67 \mathrm{p}$.

PoujaRdieu B., VISSAC B., r968. Étude biométrique de la valeur bouchère de veaux croisés Charolais et Limousin. Ann. Zootech., 17, I43-I 58.

Preston T. R., Willis M. B., 1970. Intensive beef production. Pergamon press, Oxford, p. 567. ROBERTSON A., I959. The sampling variance of the genetic correlation cofficient. Biometrics, $15,469-485$. 
SEITZ J. L., I972. Variations génétiques des difficultés de vêlage en race pure et en croisement entre trois races à viande. Dipl. Et. Appr. Agron. Montpellier, ir6 p.

SYRSTAD O., 1970. Estimating direct and correlated response to selection: a note of clarification. Acta Agv. Scand., 20, $205-206$.

TAYLOR St C. S., I968. Genetic variation in growth and development of cattle. In " Growth and development of Mammals ". Lodge and Lamming, Butter-worths, London, $527 \mathrm{p}$.

Vissac B., I964. Méthode de détermination de l'index génotypique des taureaux d'insémination sur la valeur de leurs veaux de boucherie. Ann. Zootech., 13, 267-275. 